\section{The Materials Science of Cosmetics}

\section{Steven Schnittger and Moitreyee Sinha, Guest Editors}

\begin{abstract}
The cosmetics industry is increasingly using sophisticated materials and technologies in their products and to measure their effectiveness. Understanding how these products interact with skin, adhere to skin, and deliver a benefit or create a visible effect leads to better and safer products. An understanding of the optics and mechanics of skin and the cosmetics-skin interface is important for achieving various visual and functional effects. This issue reviews the underlying science and the technical challenges behind the development of materials for the personal care and cosmetics industry.
\end{abstract}

\section{Introduction}

Cosmetics have existed since the origin of civilization. However, it is only in the last few decades that the field of cosmetics has emerged as a major scientific area overlapping a wide range of disciplines. The primary driver for this has been the development of functional cosmetics that are intended not only to improve the appearance but also to benefit the target, whether it is the skin, the hair, or the teeth. ${ }^{1}$

Today, many cosmetic products aim at hydrating the skin, reducing or slowing the signs of aged skin, or protecting the skin against the multitude of daily aggressions that it encounters. With this functional approach, products have become more diversified and require raw materials that are more efficacious, safe, bioavailable, and innovative. ${ }^{2}$ With continuous advances in the basic sciences and the development of new interdisciplinary sciences (e.g., molecular biology, biomimetics, nanotechnology), new sources for raw materials are being identified. The raw materials for cosmetic products not only are gathered from natural sources, but also can be specially synthesized or even produced from genetically manipulated microorganisms.

The influence of advances in other areas of technology is apparent in the area of cosmetics. Liposomes, microparticles, microsponges, and nanoparticles, for example, have been translated from other

Figure 1. The human skin model. most appropriate vehicle forms a research field by itself. ${ }^{3}$

There has been enormous progress in the areas of dermatology and fundamental cutaneous biology over the past two decades. ${ }^{4}$ Along with this there has been rapid development in techniques to assess aging skin by noninvasive techniques that measure such parameters as the mechanical properties of the skin, cutaneous vasculature and vasoreactivity, physical and biochemical surface characteristics, permeability, and barrier function. A major part of the visible alterations associated with skin aging reflects functional changes, that is, modifications in the various tissue components within the skin. For example, the emergence of deeper furrowing and wrinkling is related to a mechanical failure as shown by loss of tension and elasticity. The alteration of complexion is related to changes in skin vasculature. The reduction in the waterholding capacity of the outer layer of the epidermis, the stratum corneum (see Figure 1) also influences the general aspect of the skin.

With the growth of understanding of these phenomena and the development of noninvasive in vivo methods to measure these mechanisms, there has been an explosion of new materials and technology to provide functional benefits to the skin. ${ }^{2}$ Instrumentation and methodology are now available to evaluate and measure cutaneous elasticity, topography, hydration, or even to see directly in vivo inside the skin through microscopic advances.

Today, cosmetology has become a science that draws upon various disciplines, including chemistry, physics, biology, bioengineering, dermatology, microbiology, toxicology, and statistics. Because of the complexity of cosmetics science, we will

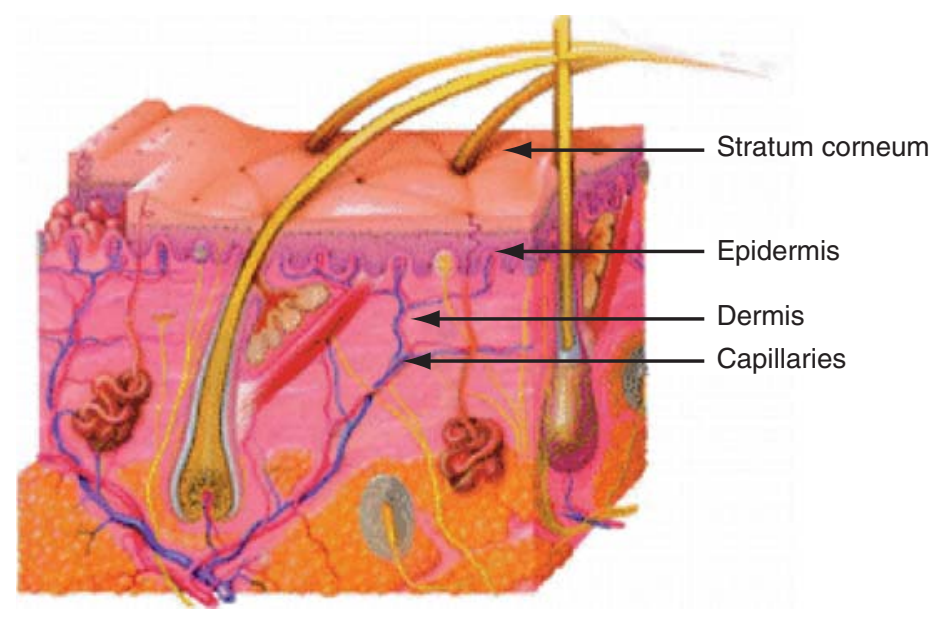


not attempt to cover all the aspects in this issue. Instead, we will focus on a few key areas to provide a glimpse into the materials science aspects of the skin, the challenges of designing materials that interact with a dynamic, complex substrate like the skin, and how these drive the development of new materials and technologies that can provide novel functionality in application.

\section{Skin: A Challenging Material Substrate}

The human skin is an extremely complex and dynamic substrate whose function is to protect against the penetration of undesirable substances. ${ }^{5}$ The skin can be the primary route of potential exposure to toxicants such as ultraviolet rays, environmental pollution, and cigarette smoke. The skin is structured in three layers: the epidermis, the dermis, and the subcutaneous layer. The outer layer of the epidermis, the stratum corneum, covers the entire outside of the body and only contains dead cells, which are strongly keratinized.

In order to develop a scientific approach to the interaction of topical applications with the skin with respect to its barrier functions, it is useful to think of the skin as a multilayer biomembrane with certain absorption properties. Beyond the stratum corneum, skin is a dynamic living tissue, so its absorption characteristics are susceptible to constant change. Additionally, it changes as a result of its own growth patterns, and this change is affected by many external factors. ${ }^{6}$ Upon coming into contact with the skin, a compound penetrates into the stratum corneum and may reach the epidermis, dermis, and the vascular network. The stratum corneum provides its greatest barrier function against hydrophilic compounds, whereas the epidermis is resistant to highly lipophilic compounds.

A reaction to an allergen may be induced by absorption of allergenic particles into the skin at the point of contact or through systemic exposure by some other means of absorption. While absorption depends on a variety of factors, such as skin hydration, body temperature, and permeability of the ingredients, the size of the molecule is considered to be the main factor in penetration. Other factors that play a role in skin penetration are surface characteristics, the presence of coatings, electronic charge, and dose. Components of the formulation may penetrate into the stratum corneum and interact with the lipid layer. This may result in disruption of the baseline membrane and cause faster penetration of the compounds into the stratum corneum. All of these factors are evaluated when determining the safety of a new raw material, especially small particles that have a high likelihood of penetration into the skin.

In addition to the barrier properties, the mechanical properties of skin are of interest, because they are directly related to the functional and aesthetic aspects of the skin. Elastic fibers are mainly responsible for the mechanical properties. The change in mechanical properties of the skin during aging is accompanied by loss of organization within the elastic collagen network and alterations of the basal elements. Therefore, these properties reflect the behavior of these elements and their structural organization. These include extensibility, relaxation, recovery, and modulus measurements of the skin and how these change with age. For example, the stiffness modulus of skin can decrease or increase depending on variations of the natural state of tension and the subcutaneous tissue with increase of flaccidity in skin or other alterations induced by the effect of aging.

Optically, the skin is a complex, translucent materials system with periodic surface features that consist of both topographical and color variations. The absorption and transport scattering properties of different layers of skin have been studied and modeled extensively. ${ }^{7}$ The absorption is described in terms of melanin and hemoglobin absorption proportional to the volume fractions of melanosomes and whole blood, with a slight baseline skin absorption. The dermal scattering is described in terms of the relative contributions of Mie and Rayleigh scattering due to collagen fibers. The epidermal scattering, which is affected by its keratin fibers, is sufficiently close to that of dermis (and sufficiently thin to not be critical) that dermal scattering can be used to describe skin scattering in general. Finally, the optics of adult skin are quite variable in the scattering properties, the degree of melanin pigmentation, and the amount and distribution of blood perfusion.

In designing cosmetics for aesthetics, the primary challenge is to hide defects such as wrinkles or blemishes while maintaining a natural appearance of the skin. Both the design of new particles and formulations as well as key understanding of the properties that are associated with a natural and healthy appearance have driven recent breakthroughs in this area.

\section{Designing Cosmetic Materials for the Skin: Function, Aesthetics, and Safety}

In today's marketplace, where the consumer is looking for a desired effect such as a reduction in lines and wrinkles, the removal of age spots, or long wear in a lipstick, the cosmetic formulation is considered to be a delivery system to achieve a desired effect. In order to get a better understanding of the materials aspects, we will focus on some of the challenges faced in the development of new cosmetic formulations as well as how advances in the understanding of structure and properties, surface chemistry, polymer chemistry, and nanotechnology are all coming together to drive the development of new materials that can help in this process.

Cosmetic products can be broken down into the following categories: skin care (including repair and anti-aging products), makeup (color cosmetics), hair care, and fragrances. Some of these product groups are briefly outlined below.

\section{Skin Care: Repair and Anti-Aging Products}

There are a variety of skin care products in the marketplace today. They fulfill a range of functions by either acting directly on the skin (moisturization, whitening) or delivering an active ingredient such as a sunscreen or anti-acne material. Materials science plays a key role in these formulations with the incorporation of raw materials such as titanium dioxide for sun protection, zinc oxide for colors and pigments, and silicone blends for a multitude of activities. Silicone products are used to boost sun protection factor (SPF) values and act as wetting agents, film formers, and water repellents. Film-forming polymers are used with sunscreens to impart water resistance and long wear. Another growing application from materials science is the use of nanotechnology in cosmetics. An example is the recent development of a shampoo that uses a negative charge to block dirt, oil, and debris from binding to the scalp.

\section{Makeup Products and Color Cosmetics}

Makeup products are principally concerned with beautifying and decoration rather than functionality. They are used to add color, hide skin flaws, act as a photoprotectant, and make the skin surface appear smoother. They include face powders, lipsticks, mascaras, and nail polishes. These products are made up of different pigments and blends of color. Conventional pigments that rely on reflected light at a certain wavelength are used to generate shades and colors; color is produced as a result of the outer electrons absorbing visible light and being promoted to the next higher energy level. Pigments that generate diverse colors can 
be organic, inorganic, or treated to enhance functionality. They are used to deflect light and create illusions in such a way that they will hide wrinkles and blemishes. Pigments are classified as microfine, ultrafine, or nano-sized particles. Inorganic pigments consist of titanium dioxide, zinc oxide, iron oxides, and ultramarines. These same pigment types are loaded into delivery systems or bound to silicone backbones, which again provide a new tool for the cosmetic chemist. Powders and pigments may be modified to make their surface water-repellent (hydrophobic), oil-dispersible (lipophilic), both lipophobic and hydrophobic (e.g., perfluoro treatments), or hydrophilic. Other objectives of surface treating are to reduce surface activity, improve skin compatibility, produce physical or chemical stability, and enhance pigment loading in formulas. There is also considerable work in using particle morphology, coatings, and multilayer systems to produce different optical effects including color and luster through interference and other mechanisms.

\section{Cosmetic Vehicles}

The active component in a cosmetic formulation is embedded into a matrix, the vehicle. The vehicle can have direct intrinsic functions such as cleansing, aesthetics, hydration, and protection, or aid in the delivery (targeting) of actives. However, regulations allow targeting only if no systemic, physiological, or pharmacological effect is achieved and the product has been shown to be safe. Delivering active components to the target requires the right concentrations to achieve optimal release rates and desired distributions of the active substances between the vehicle and the target site. ${ }^{2}$

Most cosmetic vehicles are anisotropic, heterophasic systems (mixtures). Emulsions, aerosols, foams, and gels are examples. The main components of emulsions are lipids (or lipophilic compounds) and water (or hydrophilic compounds). ${ }^{3}$ These two immiscible phases are combined with an amphiphilic emulsifier to form a metastable mixture. This biphasic system is analogous to skin, which also comprises both lipophilic and hydrophilic components. Emulsions can either be water-inoil or oil-in-water types and are prepared by dispersion of the internal phase in the external phase. Emulsifiers that decrease the interfacial tension between the two immiscible phases are used and the process of coalescence that tends to separate the two phases is slowed down by ionic or anionic surfactants. Gels (hydrogels and hydrophobic gels), hydrolipid dispersions, microemulsions, nanoemulsions and nanoparticles, suspensions, sprays, and sticks are other examples of vehicles combined with particles (active or functional).

\section{Nanoparticles as Carriers}

Novel active ingredients, new surfactant molecules, and novel vehicles or carriers are leading to the development of new cosmetic systems in contrast to classic forms such as creams and gels. Recently, there has been extensive research in the area of controlled delivery systems. Novel polymers and surfactants can aid in this goal. Encapsulation techniques are used to control the delivery of encapsulated agents as well as protect those agents from environmental degradation.

Recently, special dispersion formulations have been developed with ultrasmall particles used as carriers for active components. The size of the particles ranges from $10 \mathrm{~nm}$ to a few hundred nanometers. For example, solid lipid nanoparticles possess a solid matrix composed of physiological lipids with mean diameters ranging from $50 \mathrm{~nm}$ to $1 \mu \mathrm{m} .^{8}$ Active components may be incorporated into these lipid nanoparticles, which serve as carriers. Alternately, the core of the nanoparticles may either be a liquid lipid functioning as carrier or a lipophilic agent being directly effective, for example, an emollient or occlusive agent. Lipophilic active substances, such as vitamins A or E or ultraviolet filters, can be incorporated.
Lipid nanoparticles have a similar structure to nanoemulsions. Their size typically ranges from $50 \mathrm{~nm}$ to $1 \mu \mathrm{m}$. The difference is that the lipid core is in the solid state. The matrix consists of a solid lipid or a mixture of lipids. To stabilize the solid lipid particles against aggregation, surfactants or polymers are added; natural lecithins are preferred, as is the case with nanoemulsions. If lipid nanoparticles are intended to be used as a carrier, the active ingredients are dissolved or finely dispersed in the lipid matrix.

Nanoparticles can be segmented by size and structure of the encapsulating membrane. Liposomes can encapsulate and transport water-soluble ingredients in their polar cavity and oil-soluble ingredients in their hydrophobic cavity (Figure 2). These can form effective delivery systems for hydrophilic actives by providing active stability and increased skin penetration. Liposomes have been in use since the mid1980s, when Christian Dior first introduced its Capture line to the market.

Nanosomes and nanotopes differ from liposomes in that the membrane is a single layer; the polar heads are on the outside of the spheres and the tails point inward to form a lipophilic core. Nanosomes are used to carry lipophilic material. Nanotopes are formed from a combination of lecithin and cosurfactant, which are aligned in an alternating arrangement. An example of a commercialized nanotechnology product is L'Oreal's Plenitude Revitalift, introduced in 1998, an anti-wrinkle cream that uses a $200 \mathrm{~nm}$ nanotechnology
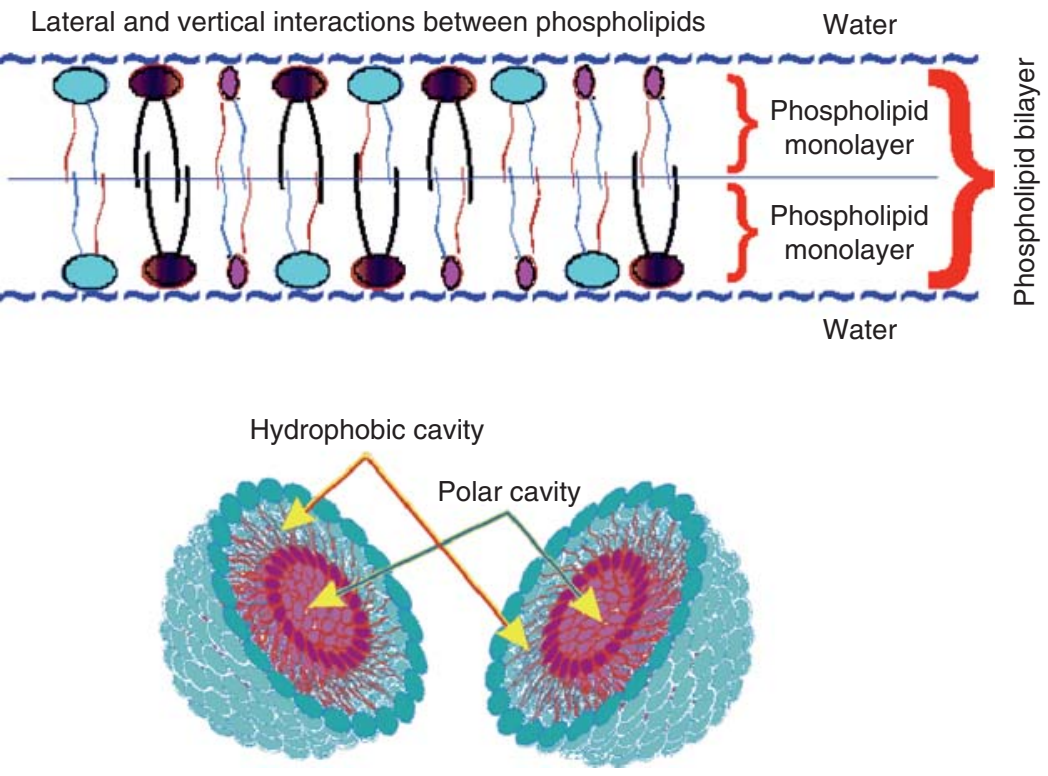

Figure 2. Liposomes can encapsulate and transport water-soluble ingredients in their polar cavity and oil-soluble ingredients in their hydrophobic cavity. 
(nanosome) process to incorporate vitamin A into a polymer nanocapsule. The nanocapsules-vesicular systems in which the active ingredient is confined to a cavity surrounded by a polymeric membrane-help deliver active ingredients to the skin's deeper layers.

Nanoemulsions are water-thin transparent emulsions (they suggest purity and cleanliness) that can be sprayed onto the skin-the particle size is small and the emulsion incorporates into the skin with little residue. Enhanced delivery of the active to the skin is thus achieved Nanospheres are matrix systems in which the active ingredient is dispersed throughout the particles.

The number of cosmetic products made using nanotechnology is rapidly increasing and is a critical area of debate with respect to safety. While recognizing the value of these molecular-level advances, there is concern about the safety of nanotechnology for workers and consumers. The advantages of nanoparticles as delivery systems include

- The solid matrix provides flexibility to modify the release profile of the active ingredient,

- The relatively slow degradation allows long release times, and

- The nanoparticles provide protection of incorporated compounds against chemical degradation.

Release mechanisms are different in nanospheres and nanocapsules. ${ }^{3}$ In nanospheres, when the active ingredient is superficially adsorbed, the mechanism is a partitioning process. When the ingredient is entrapped in a matrix, diffusion is the mechanism along with bioerosion, if the carrier is biodegradable. Release from nanocapsules is related to the partitioning processes within immiscible phases. However, many unanswered questions remain in this field. In order for a holistic picture to emerge, the materials science development needs to be accompanied by well-controlled biological and percutaneous penetration studies. ${ }^{3}$

\section{Design of Cosmetic Formulations}

Cosmetic formulations are complex and are often arrived at by trial-and-error procedures. In recent years, there has been a great demand to rationalize these compositions, clarifying their complex interactions and applying fundamental principles of colloid and interface science to these systems. Figure 3 shows a schematic representation of a multiple emulsion drop, showing the role of the various components. ${ }^{9}$ Whenever the target of an active component lies in the deeper regions of the skin, the active component must be in the molecular form for efficient delivery. If there are solubility or stability issues, then the component has to be incorporated in particulate form. Fine particles on the order of $1 \mu \mathrm{m}$ can be delivered into the uppermost layers of the skin, where they will dissolve at rates based on their solubility in the skin. The preparation and solubilization of multiphasic systems require the addition of amphiphilic substances such as emulsifiers and surfactants for wetting and repulsing particles in suspensions. The driving force for the delivery of components (i.e., release from the vehicle and penetration into skin), is a thermodynamic activity, which is maximal at saturation concentration. ${ }^{10}$ However, saturated or supersaturated systems are not the only drivers for optimal topical delivery. For example, the skin-vehicle partition coefficient of the component also plays a role. Moreover, the partition coefficient may increase due to skin-vehicle interactions, which will lead to increased skin penetration.

Sun-protection products are a good example of systems showing interactions among vehicle, active component, and the skin. ${ }^{3}$ The absorption of UV radiation depends on not only the molecular structure and concentration of the protecting agent but the solvent as well. Therefore, water resistance may be influenced by selection and composition of the vehicle. Further, vehicle components may penetrate into the skin and interact with stratum corneum lipids. This can disturb their lamellar structures, leading to increased and faster penetration of compounds into the stratum corneum. Alternatively, the presence of vehicle components in the stratum corneum may cause a depot effect for certain compounds.

Another key materials aspect that affects the performance of a formulation is the substantivity, which is a measure of the adherence of materials to keratinous substrates in the upper skin layers. ${ }^{11}$ This is primarily a function of the active molecules but may also be influenced by the vehicle. For example, the addition of filmforming, skin-adherent polymeric substances to the vehicle may increase the retention of sunscreens in the skin and result in a product with improved waterresistance. Another example is formulations with phospholipids, which form vesicular, liposomal structures in the vehicle or in the upper layers of the stratum corneum, yielding a depot effect.

At certain concentrations and combinations of specific emulsifying agents, liquidcrystalline structures called mesophases are formed. ${ }^{3}$ These show anisotropy and are thermodynamically stable. This can significantly impact the behavior of a vehicle. For example, fatty amphiphiles that are dispersed in water in the presence of a high hydrophilic-lipophilic-balance surfactant form lamellar phases. These are capable of swelling at elevated temperatures close to the melting point of the hydrocarbon chain. These swollen lamellar liquid-crystalline phases can incorporate significant quantities of water. With a

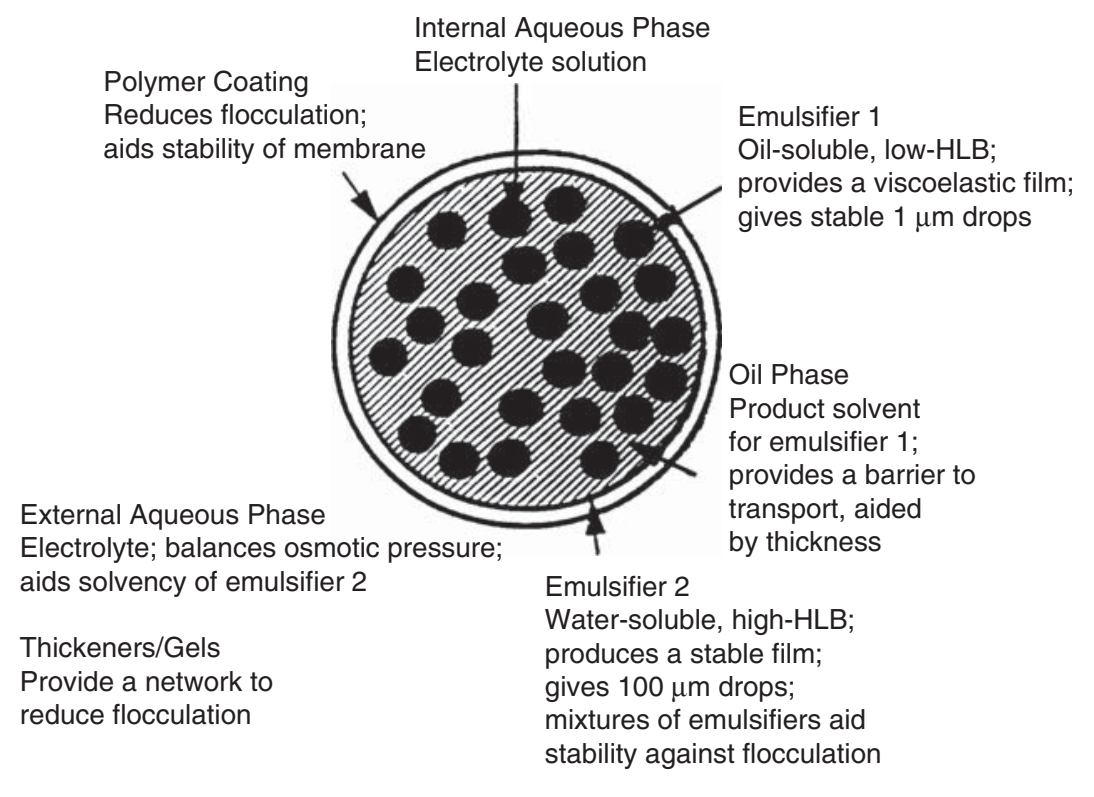

Figure 3. Schematic representation of a multiple emulsion drop, showing the roles of the various components. HLB is hydrophilic-lipophilic balance. ${ }^{9}$ 
decrease in temperature, the disordered liquid-like chains transform to form lamellar crystalline-gel network phases. Such networks not only stabilize creams and lotions but also control their consistency due to their viscoelastic properties. Such mesophases provide key advantages to emulsions, including increased stability, prolonged hydration properties, controlled release of active ingredients, ease of formulation, and good "feel" on the skin.

Most vehicles undergo considerable changes during and after application to the skin, owing to the mechanical stress caused when the formulation is spread over the surface and as volatile phases evaporate. Mechanical stress and skin temperature may influence the viscosity of the vehicle and consequently the release rate of active ingredients. Uptake of water from the skin may alter the composition of the vehicle. All of these factors may also cause phase inversion or phase separation. This can alter the thermodynamic activity of an active ingredient. Thus, by controlling or altering the thermodynamic activity, release of a component and its penetration into skin can be modulated. For example, if after application, the volatile phase evaporates, saturation or supersaturation concentrations of the active component can be reached. This results either in improved release and delivery or in precipitation and deposition of the active component. Another example is optimally composed sun-protecting oilin-water emulsions. After application, the emulsion transforms to a water-in-oil type due to evaporation of water and the mechanical stresses caused by spreading. The remaining lipophilic protective film leads to improved water resistance.

In summary, the optimal design of vehicles for ease of manufacturing and storage and for balancing properties after application and metamorphosis on a complex substrate like the skin is a rich scientific field.

\section{Dermal Absorption and Safety Testing}

The skin presents a barrier, both from a structural and chemical composition perspective. Dermal absorption is the process by which a substance is transported across the skin and taken up into the living tissue of the body. Dermal absorption of a cosmetic product is influenced by many factors, such as its liposolubility, the molecular weight and concentration of the particles in it, the vehicle used to deliver the product, whether the skin is occluded, the exposure pattern, and the skin site of the body. For example, Figure 4 shows the effects of the vehicle on skin absorption in a study where lidocaine was applied to the skin in an in vitro absorption study. ${ }^{6}$ Diffusion across the skin membrane is regulated by Fick's law, which states that the rate of diffusion across the barrier will be directly proportional to the concentration gradient.

As new products are brought into the marketplace, their safety and stability are thoroughly evaluated long before their release to the consumer. Cosmetic products that affect the structure or function of the human body are considered by the FDA to be drug products and are regulated as such. Examples of over-the-counter (OTC) products are toothpastes, sunscreens, antibacterial soaps, and anti-perspirants. A product may be a cosmetic, a drug, or both. Cosmetic products are typically brought to market very quickly, because the window of marketing opportunity for new products is short-lived. FDA approval is not required to bring new cosmetic products to market, and unless there is a consumer complaint, the FDA does not have regulatory control over cosmetics.

The manufacturer of a cosmetic product is responsible for the safety of the product and its ingredients. Products must be formulated to minimize the possibility of irritation and allergic response. Even minor disturbances of the skin surface can produce discomfort, especially in the facial area, which has an extensive network of
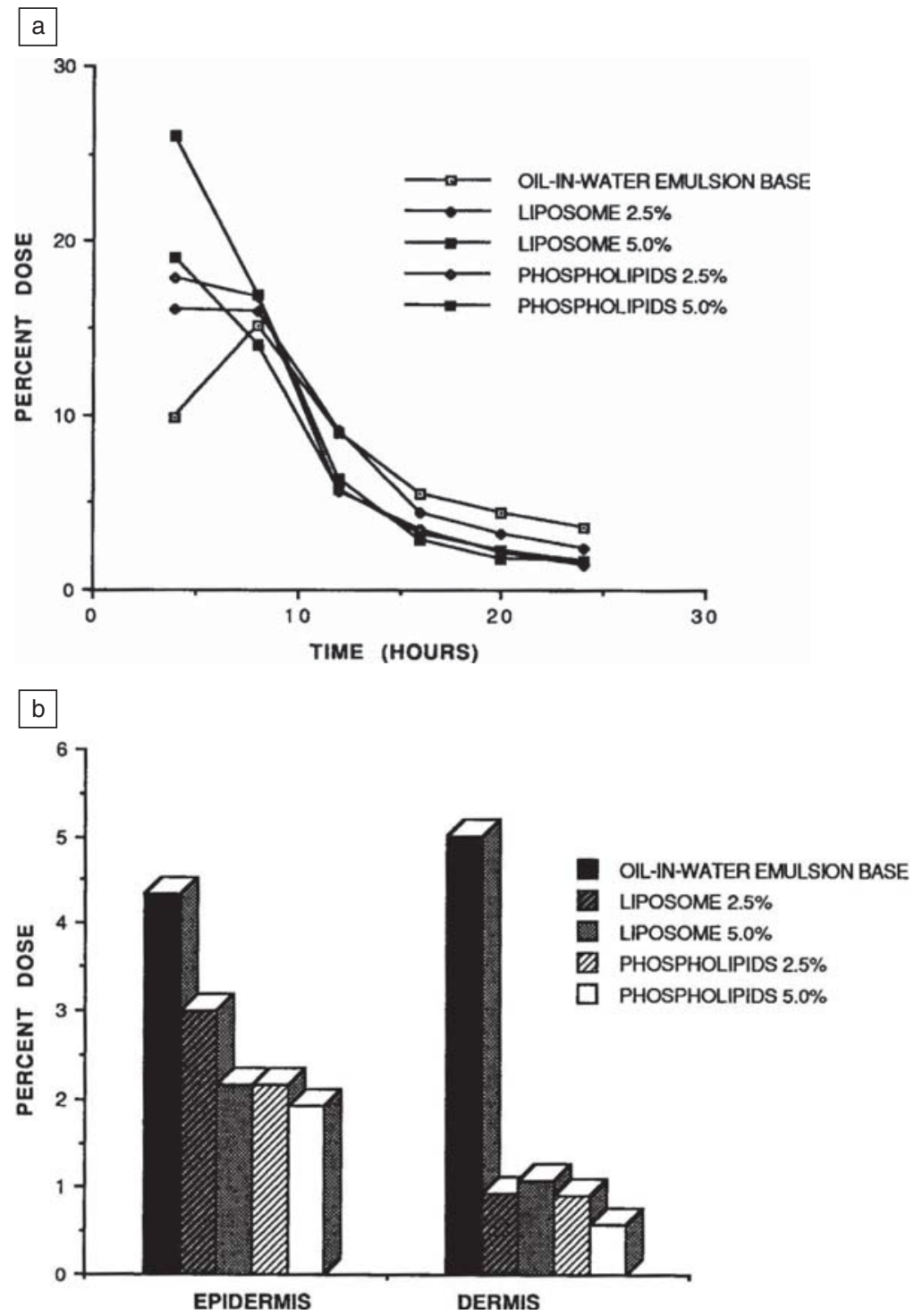

Figure 4. (a) Lidocaine percutaneous absorption through human skin. Formulation determines the initial absorption. (b) Distribution of lidocaine in human epidermis and dermis. Formulation determines the concentration within the skin component. ${ }^{6}$ 
sensory nerves. Any new raw material now delivered to the marketplace must go through extensive testing to ensure that it is safe as recommended. This increase in safety requirements is due to increased regulatory requirements, especially in the European Union, where the REACH program (Registration, Evaluation, Authorization, and Restric-tion of Chemicals) requires cradle-to-grave documentation for all raw materials.

Numerous microbiological, toxicity, and safety tests are performed to ensure the safety of a cosmetic product. These different types of tests occur at all stages of development, from the raw materials to small lab batches $(\sim 1 \mathrm{~kg})$ to pilot batches $(\sim 10 \mathrm{~kg})$ to production requirements (1000 $\mathrm{kg}$ and above). Safety testing includes both in vitro laboratory testing and largescale clinical in-use studies to prove both product concept and safety in use.

In order to test for dermal absorption of a particular compound, different techniques are used, since a new compound may be potentially toxic and cannot be tested directly on humans. Two major methods are in vitro cell techniques and in vivo determinations. Justification for the use of in vitro studies on isolated skin is based on the fact that the epidermis forms the principal in vivo barrier. The in vitro dermal tissue may retain compounds that in vivo may be carried away into systemic compartments; therefore, possible in vitro retention in the dermis must be taken into account when interpreting the penetration data.

With the recent introduction of nanotechnology to cosmetics, there is the concern that the tiny particles of these low-molecular-weight compounds may penetrate the skin barrier, which may lead to allergic or more severe reactions. Examples of actives are high-purity zinc oxide nanoparticles for UV protection combined with a very high degree of transparency. These spherical zinc oxide nanoparticles are approximately $20 \mathrm{~nm}$ in diameter with narrow particle size distributions, while retaining their discrete nonaggregating properties. Zinc oxide products are available as dry powders or stable dispersions in a variety of solvents, both aqueous and organic.

Penetration into intact skin is in part dependent on particle size, meaning that skin uptake of smaller particles is more likely than of larger particles. There is a general relationship between particle size and toxicity: the smaller a particle, the greater is its surface-to-volume ratio and potential toxicity, but these results are not always clearly defined. There are conflicting results to this theory based on the abil- ity of zinc oxide and $\mathrm{TiO}_{2}$ nanoparticles to cross the skin membrane. The conflicting results are related to the methods used to measure the penetration of the nanoparticles into the skin. There is some concern whether the industry has the right technology to properly measure the penetration of these particles into the skin and therefore to determine if there is the potential for an adverse reaction. It has been reported that micrometer-sized particles of $\mathrm{TiO}_{2}$ were able to get through the stratum corneum, but this effect was not observed as penetration since it was clustered around a hair follicle. ${ }^{12}$ For most chemicals, the stratum corneum is the rate-limiting barrier to percutaneous absorption and penetration. Most studies concerning penetration of materials into the skin have focused on whether drugs penetrate the skin, using different formulations containing chemicals or particulate materials. In a recent review, Kreilgaard argued that very small $\mathrm{TiO}_{2}$ particles penetrate into the skin and can interact with the immune system. ${ }^{13}$ Tinkle et al. demonstrated that $0.5-1.0 \mu \mathrm{m}$ particles penetrate the stratum corneum. ${ }^{14}$ With the focus on materials science within the cosmetics industry, the issue of what type of reactions will occur if these particles penetrate into the dermis is constantly being reviewed.

\section{Irritation and Sensitization Testing}

All products and materials must be tested for skin irritation and sensitization, and any product used around the eye must also be evaluated for potential eye irritation. Skin reactivity varies, depending on the anatomical site. Variations in skin reactivity are attributed to differences in keratinization and in the density of epidermal shunts such as hair follicles and sweat ducts. Many cosmetics companies manufacture products that are considered hypoallergenic by focusing on raw materials that have the lowest possibility of being an allergen.

The HRIPT, or human repeat-insult patch test, is the most commonly used method of evaluating product sensitization in vivo now that animal testing has been banned from use. It involves applying the test formulation on the skin for a known period of time and inspecting the skin for irritation, redness, or blistering. Photosensitization is evaluated in the same manner by exposing the test area to low doses of UV radiation. With an increased focus on consumer safety and increases in regulatory requirements, all raw materials are now required to have a minimum HRIPT performed before use in a product.

\section{Toxicity Testing}

For cosmetics products intended for topical application to the skin, safety issues include irritation, sensitization, toxicity, and carcinogenicity. A further issue for topical products is their potential interaction with UV radiation to produce photo-induced irritation, sensitization, toxicity, or carcinogenicity. Prior to the release of new products, in vitro and in vivo tests are often conducted in order to screen for safety and efficacy. There are currently a number of rapid and reliable in vitro methods that have replaced the testing of animals for safety screening of cosmetic formulations. Over the past decade, advances in culture methodology, including the development of defined media, matrices, and effective growth factors, have brought cell and tissue culture to new levels of sophistication.

An example of an advance in tissue culture methodology is three-dimensional organotypic skin models, which are cultured at the air-liquid interface and are suitable for safety and efficacy testing of topical products. Among the safety issues that organotypic skin models can address are tissue damage (i.e., cell death by necrosis or apoptosis), irritation, and DNA damage caused by topical treatment of UV radiation. Genotoxicity and mutagenicity studies on raw materials are also carried out and any indications of potential effects require elimination of the use of these raw materials.

As materials science and nanotechnology expand within the cosmetics industry, advanced methods for measuring dermal absorption and determining the potential risks must be developed.

\section{Microbiological Testing}

Most cosmetic products contain water and therefore have the potential to support microbial growth. There are a number of ways this is addressed: addition of an antimicrobial agent, sterile and aseptic production and filling into packaging material, and reduced water activity. If bacteria have the ability to grow to high numbers, there is the potential for product spoilage and a health hazard to the consumer. Because of this risk potential, most products require the addition of a preservative to the formulation to help protect against microbial contamination and ensure longterm stability. The materials science aspects of adding preservatives to complex, multiphasic systems form a critical area in formulations. It is mandatory not only to add antimicrobials but also to test their efficacy after manufacturing and storage until the expiration date. Nowadays, performance of the preservative efficacy test is standard 
practice. At each stage of development and manufacturing, both raw materials and finished products are checked for microbial content. Stability programs are also put in place to ensure long-term stability of the product and its major ingredients.

\section{Assessing Performance on Skin: Sensory Assessment and Consumer Use Studies}

Until recently, sensory assessment using expert panels was the main technique used by the cosmetics industry for evaluating visual or sensory performance on the skin. Although it is a very subjective method, it remains an important tool, and valuable data is obtained if these panels are carried out in a systematic way. With advances in instrumentation, statistical methods, and a fundamental understanding of skin-formulation interactions, cosmetics companies are using increasingly sophisticated technologies to measure the effectiveness of their skin care products.

The measurement of human perception remains a challenge, however, and there is a need for objective methodologies that allow the quantification of the visual appearance of lines and defects on skin. ${ }^{15}$ For example, there are several approaches to reduce the appearance of lines and wrinkles ranging from using oxidants and moisturizers to slow down their formation, chemical peels to reduce wrinkle depth, and light diffusion to lower the visibility of wrinkles. The human perception of lines and wrinkles on skin is, however, independent of the chemistry and mechanisms used to reduce their appearance. For lines and wrinkles, optical imaging of skin is becoming a critical tool for assess- ment, since it allows the measurement of spatial contrasts that the human eye is most sensitive to (Figure 5). Unlike expert panel assessments, these laboratory-scale methods can be used to accelerate the materials development earlier in the process by screening a range of particles, vehicles, and formulations. In addition, since these measurements can be directly correlated with the properties of materials, skin, and their interactions in a systematic way, a deeper insight into the mechanisms can be obtained, leading to smarter design of materials.

To use these techniques, however, for developing predictive capabilities for the complex process of visual perception, it is important to understand the fundamental interactions of visible light with surfaces and defects to form contrasts, as well as the human sensitivity to different optical attributes of surfaces, defects, and their interactions. A limitation of these objective assessments is that they often measure only one aspect of skin aging. Therefore, a number of techniques have to be combined in the right way to correlate with human perception. The reliability and precision of such psychosensorial assessments depend on the use of appropriate standards, scales, and optical imaging. This is not an easy problem, and therefore, there continues to be a need for overall skin aging assessments.

\section{In This Issue}

The articles in this issue of MRS Bulletin review the underlying science and the technical challenges behind the development of materials for the personal care and cosmetics industry.
Subramanyan et al. introduce the materials properties of the stratum corneum, from the molecular to mesoscale assembly of hierarchical structures. The ability of skin to respond to day-to-day challenges such as changes in temperature and humidity is examined from a materials science and biophysical perspective.

Delivery of active ingredients can be enhanced through the use of liposomes, which improve lipid solubility and penetration through the skin. In their article, Somasundaran et al. discuss how nano-sized particles, hybrid polymers, and encapsulates are incorporated into cosmetic products to improve this delivery process. The benefit of liposome technology is in the ability of liposomes to stabilize active ingredients in their native form by encapsulation, thus slowing the degradation process that may be accelerated in the presence of oxygen or UV light. They also discuss the safety aspects of nanomaterials for cosmetics and the need for the development of toxicological methods to assure nanomaterial safety.

Maitra and Brahms discuss the biomechanical, surface, and transport properties of the skin. They focus on the challenges faced in the development of new formulations in color cosmetics, such as long wear, appearance, and comfort, and how advances in the understanding of materials properties are leading to the development of new materials.

In their article, Cummins and Fthenakis discuss tools such as confocal laser scanning microscopy (CLSM) and scanning probe microscopy (SPM), which are used to measure the physical and optical prop-
Skin
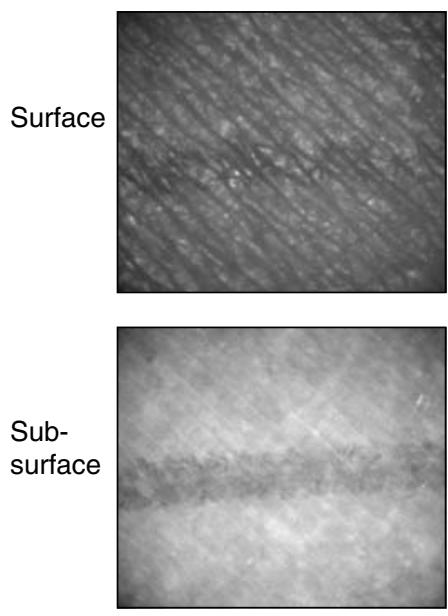

Product A
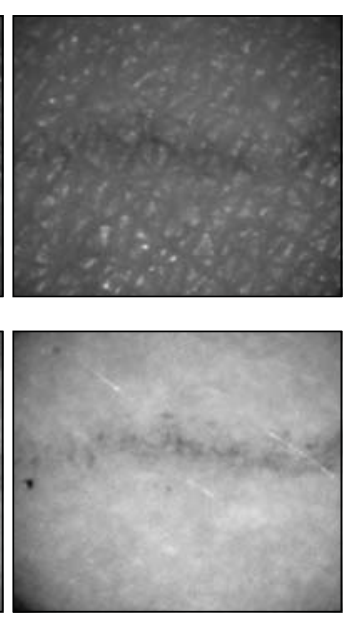

Product B
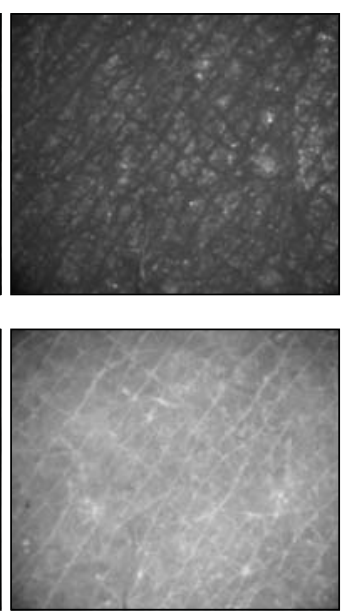

Product $\mathrm{C}$
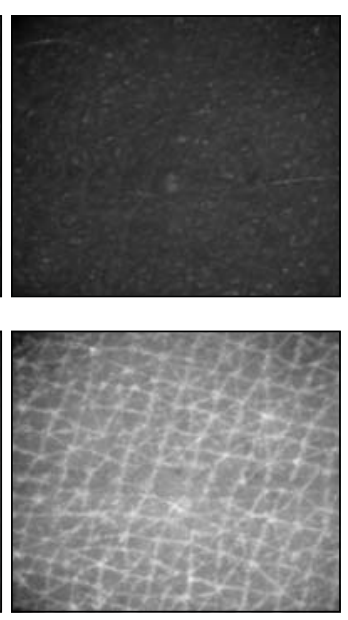

Product D
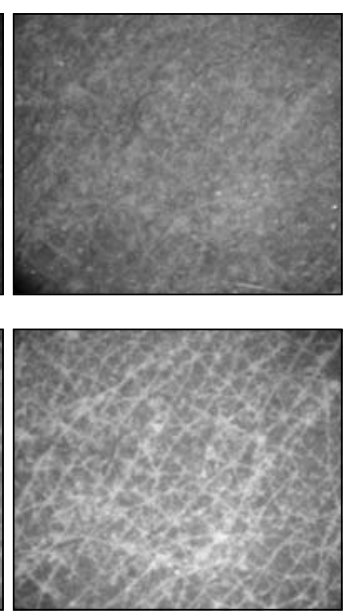

Figure 5. Surface and sub-surface light scattering on skin measured using optical imaging that shows the differences in appearance based on the nature of the product applied to the skin. ${ }^{16}$ 
erties of the skin. The ability to quantitatively measure these parameters, and the understanding it provides on how the skin interacts with product formulations, are of critical importance to the development of a cosmetic product.

In the last article, O'Lenick and O'Lenick discuss the increase in the use of silicone polymers and their importance in cosmetic products. Silicones, when properly chosen, can provide to formulations surface tension reduction, wetting, emulsification, and film-forming properties.

\section{Conclusion}

There is no one cosmetic formulation or vehicle that will fit all requirements. Every cosmetic formulation must be built for a desired effect or application. In this issue, we have tried to present a window into some of the aspects of developing a cosmetic product and what it takes to bring that product to market. It is our hope that we have provided a small snapshot of the complexity of product development and how the cosmetics industry taps other industries for fresh ideas and new ingredi- ents to improve product efficacy. The field of materials science is one of those avenues that has recently been tapped for the next new breakthrough in cosmetics science.

\section{References}

1. E.D. Goddard, J.V. Gruber, Eds., Principles of Polymer Science and Technology in Cosmetics and Personal Care, Cosmetic Science and Technology Series, vol. 22 (Marcel Dekker, New York, 1999). 2. A.O. Barel, M. Paye, H.I. Maibach, Eds., Handbook of Cosmetic Science and Technology (Marcel Dekker, New York, 2001).

3. S. Buchmann, "Main Cosmetic Vehicles," in Handbook of Cosmetic Science and Technology, A.O. Barel, M. Paye, H.I. Maibach, Eds. (Marcel Dekker, New York, 2001).

4. J. Leveque, P.G. Agache, Eds., Aging Skin (Marcel Dekker, New York, 1993).

5. J. Elias, in Handbook of Cosmetic Science and Technology, A.O. Barel, M. Paye, H.I. Maibach, Eds. (Marcel Dekker, New York, 2001) pp. 19-27. 6. R.C. Wester, H.I. Maibach, in Handbook of Cosmetic Science and Technology, A.O. Barel, M. Paye, H.I. Maibach, Eds. (Marcel Dekker, New York, 2001) pp. 53-65.

7. For example, I.S. Saidi, S.L. Jacques, F.K. Tittel, Appl. Opt. 347410 (1995); S.L. Jacques, Appl. Opt. 322447 (1993).
8. J. Jansen, H.I. Maibach, in Handbook of Cosmetic Science and Technology, A.O. Barel, M. Paye, H.I. Maibach, Eds. (Marcel Dekker, New York, 2001) pp. 171-190.

9. Th.F. Tadros, in Principles of Polymer Science and Technology in Cosmetics and Personal Care, vol. 22, E.D. Goddard, J.V. Gruber, Eds. (Marcel Dekker, New York, 1999) pp. 73-112.

10. G.L. Flynn, N.D. Weiner, in Dermal and Transdermal Drug Delivery, R. Gurny, A. Teubner, Eds. (Wissenschaftliche Verlagsgeschellschaft, Stuttgart, 1993) p. 44.

11. U. Hagedorn-Leweke, B.C. Lippold, Eur. J. Pharm. Biopharm. 46, 215 (1998).

12. J. Lademann, H. Weigmann, C. Rickmeyer, H. Barthelmes, H. Schaefer, G. Mueller, W Sterry, Skin Pharmacol. Appl. Skin Physiol. 12, 247 (1999)

13. M. Kreilgaard, Adv. Drug Deliv. Rev. 54 (Suppl. 1), S77 (2002).

14. S.S. Tinkle, J.M. Antonini, B.A. Rich, J.R Roberts, R. Salmen, K. DePree, E.J. Adkins, Environ. Health Perspect. 111, 1202 (2003).

15. G. Grove, M.J. Grove, in Handbook of Cosmetic Science and Technology, A.O. Barel, M. Paye, H.I. Maibach, Eds. (Marcel Dekker, New York, 2001) pp. 829-835.

16. M. Sinha, Proc. 23rd. Congr. Int. Fed. Societies of Cosmetic Chemists (Orlando, Fla., 2004) pp. 289-295.

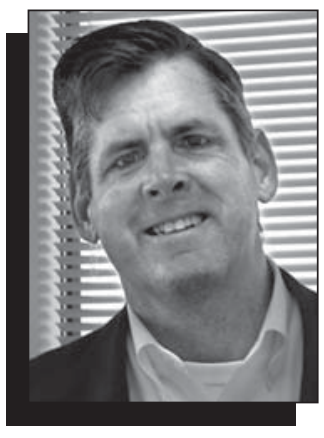

Steve Schnittger

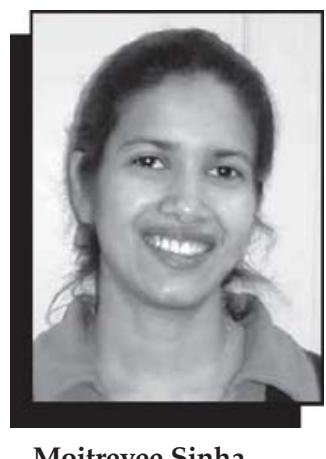

Moitreyee Sinha
Steve Schnittger, Guest Editor for this issue of MRS Bulletin, is executive director of Microbiology—Research and Development for the Estée Lauder Companies. Schnittger has been with Estee Lauder for more than 20 years. His primary responsibility is cosmetic preservation and advising Lauder chemists on new formulations. Other major responsibilities include developing new active ingredients through fermentation.
Before coming to Estée Lauder, Schnittger worked for Ralston Purina Co. and attended the University of Tulsa, majoring in microbiology.

He is a past chairman of the CTFA Microbiology Committee and a member of the American Society of Microbiology, the Society of Cosmetic Chemists, and the Institute of Food Technology. Schnittger has given frequent presentations around the world on cosmetic preservation and holds many patents in the field of cosmetic preservation and active ingredients for use in cosmetics.

Schnittger can be reached at Estée Lauder Companies, 125 Pinelawn Rd., Melville, NY 11746, USA; tel. 631531-1521, fax 631-5015803, and e-mail sschnitt@estee.com.

Moitreyee Sinha, Guest Editor for this issue of MRS Bulletin, is a senior research scientist at the Optical Polymers Laboratory in General Electric's Global

Research headquarters in Niskayuna, N.Y. She completed her master's degrees at the Indian Institute of Technology, Kharagpur, followed by a $\mathrm{PhD}$ in physics from the University of Cincinnati. She joined GE Research in 2000 and pioneered an area of optical technology to quantify visual perception of surface aesthetics.

In recent years,

Moitreyee has been

leading programs over a wide range of technical areas including new materials and optical methods for skin care technology, coatings for optical media technology, fire-retardant plastics for aircraft interiors, and new applications using holography. She is a recipient of GE's Dushman Award, holds 10 patents, has published nine papers, has given 24 invited talks, and coauthored a book chapter.

Moitreyee can be reached at Polymer and Chemical Technology, CEB-2597, GE Global Research, Niskayuna, NY 12309, USA; tel. 518-5338238 and e-mail sinha@crd.ge.com.

K.P. Ananthapadmanabhan is currently a group project leader in the

Materials Science Group of Unilever Research and Development in Trumbull, Conn. Ananth received his BTech degree from the Indian Institute of Technology, Mumbai, and his mas- ter's (1976) and doctorate (1980) degrees from Columbia University. He was a member of the Surface Chemistry Skill Center of Union Carbide Corp. from 1983 to 1990, before joining Unilever $R \& D$ in 1990, where he is now leading a group on surfactants and polymers. His research interests include surfactant solution chemistry, surfactant interactions with polymers, proteins, biomembranes and skin.

Ananth has co-edited a book on the interactions of surfactants with polymers and proteins and has more than 20 patents and over 80 publications.

Ananth can be reached by e-mail at kp.ananth@ unilever.com.

John C. Brahms is the director of research and development at Coughlan Products Corp. in Wayne, N.J. He earned his BS degree in chemical engineering from Drexel University and his $\mathrm{PhD}$ 


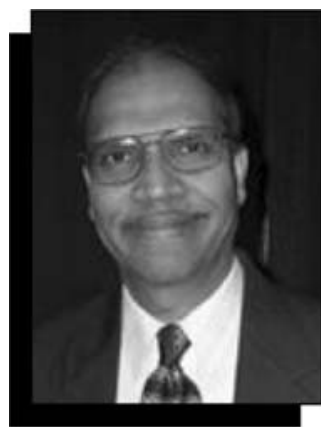

Ananthapadmanabhan

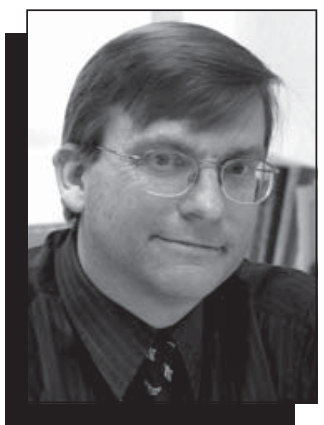

John C. Brahms

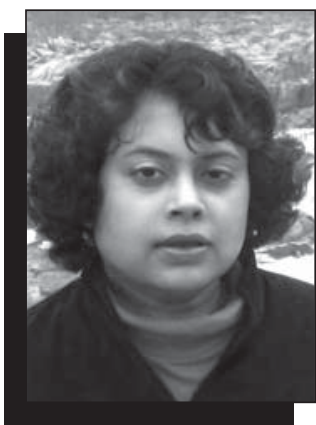

Soma Chakraborty

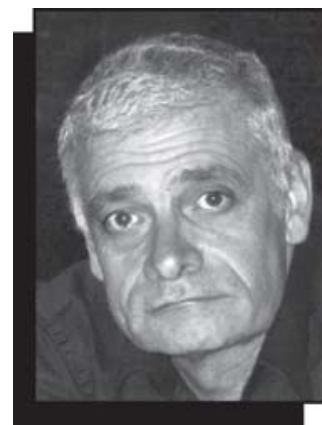

Phillip G. Cummins

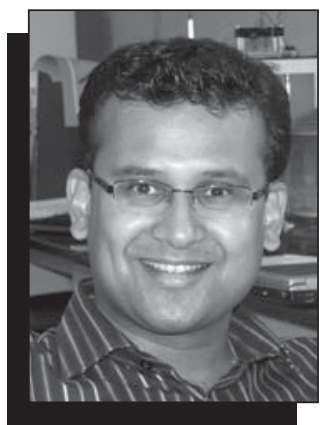

Prithwiraj Maitra degree in physicalorganic chemistry from the University of Pennsylvania. Brahms held several positions of increasing responsibility with Colgate Palmolive Co., then Avon Products Corp. His research interests include applying a multidisciplinary approach to developing novel products. He is a co-inventor on 11 granted patents, with eight pending applications. Brahms has authored 16 peerreviewed publications, including three book chapters. He is a member ACS and AIChE.

Brahms can be reached at Coughlan Products Corp., 357 Hamburg Turnpike, Wayne, NJ 07470, USA; tel. 973-9041500 ext. 32, fax 973-9049191, and e-mail johnb@ coughlanproducts.com.

\section{Soma Chakraborty is an assistant professor at Ateneo de Manila University in Manila, Philippines. She received her BS degree from Osmania University, her MS degree from the University of Hyderabad, her MTech degree from the Indian Institute of Technology, and her PhD degree from Polytechnic University in New York. Chakraborty was a post- doctoral research fellow at Columbia University from 2003 until she joined Ateneo de Manila University in 2005. Her Measurement Science for the company. Cummins moved to Estée Lauder in 1998. \\ Cummins can be reached by e-mail at pcummins@estee.com. \\ Christina Fthenakis is director of Material Sciences at Estée Lauder Research \& Development in Melville, N.Y. \\ Prithwiraj Maitra is a senior scientist in the New Technology Department at Avon Product Company's Global Research facility}

research interests include biocatalysis-controlled release vehicles using biopolymers.

Chakraborty can be reached by e-mail at schakraborty@ateneo. edu.

Phillip G. Cummins is the executive director for material science and polymers for Estée Lauder Companies. He trained in the dielectric and optical properties of liquid crystals at the University of Sheffield and also in the spectroscopic behavior of small gaseous molecules at University of Southern California and MIT. Cummins joined Unilever Research in 1979, where he developed his skills in the physical measurement of fluids and colloid systems, finally heading up the Department of in Suffern, N.Y. He received his $\mathrm{BS}$ degree in chemistry and his BTech degree in polymer/paint technology from the University of Bombay, India. In 2003, Maitra received his $\mathrm{PhD}$ degree in polymer/physical chemistry from Temple University. He performed his postdoctoral work at Colgate Palmolive Co. in the Oral Care Division. Maitra's current responsibilities include exploration and discovery of new materials and technology for application in color cosmetics and skin care. He has authored seven peer-reviewed publications, holds one patent, and has six pending patent applications. He recently received Avon's Global R\&D Achievement Award for contributions towards developing novel optical effects.

Maitra can be reached at One Avon Place, Suffern, NY 10901, USA; e-mail prithwiraj.maitra@ avon. com.

Somil C. Mehta is a graduate student working toward his $\mathrm{PhD}$ degree at the Department of Earth and

Environmental

Engineering at Columbia University.

Manoj Misra leads the Microstructure Imaging Group for Products and Skin at Unilever
Research and

Development in

Trumbull, Conn. He is mainly interested in utilizing various imaging modalities to determine the relationship between microstructure and function of product films and their effect on the microstructure of skin. Misra obtained his PhD degree in physics from the University of Alberta, studying radiation damage in biomolecules. He did postdoctoral research at Duke Medical Center. Subsequently, Misra worked as a scientist at the Skin Research Institute of the National Institutes of Health before joining Unilever. His work there has centered on exploiting imaging techniques to quantify ultrastructural water distribution in skin and assess the role of crystalline packing and cell coverage of skin lipids on skin health. Misra is also interested in improving the understanding of underlying causes of skin pigmentation and the effect of photo- and hormonal aging on skin.

Misra can be reached by e-mail at manoj. misra@unilever.com.

Surajit Mukherjee is a lead scientist within the Materials Science Group at Unilever Research and Development in Trumbull, Conn. He

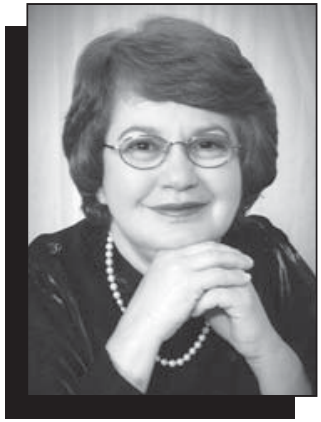

Linda D. Rhein

received his $\mathrm{PhD}$ degree in chemical engineering from Carnegie Mellon University, working in the area of microemulsions. Mukherjee did postdoctoral research in micelles at the University of Minnesota before joining Exxon Chemical Co., where he worked on crude oil demulsification.

Mukherjee has been with Unilever R\&D since 1988, where he has led research groups in skin biophysics, dermal delivery, and new skin improvement devices. His current research interests are developing technologies for novel skin sensory benefits and understanding of the physics of deposition from cleansers.

Mukherjee can be reached by e-mail at surajit.mukherjee@ unilever.com.

Anthony J. O'Lenick is president of Siltech LLC, a company he founded in 1989. Siltech is a silicone 


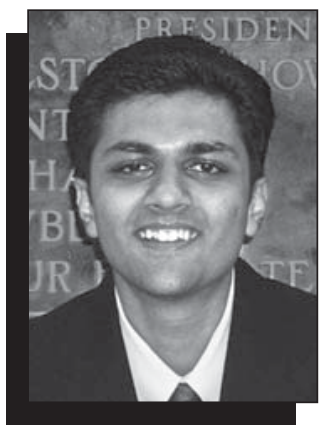

Somil C. Mehta

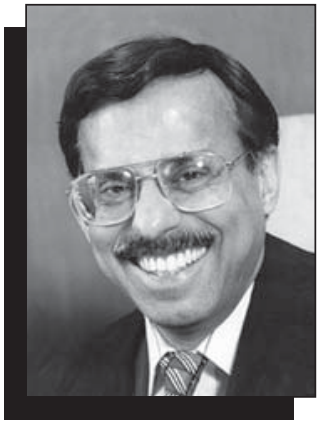

P. Somasundaran

and surfactant specialty company. Prior to Siltech, O'Lenick held technical and executive positions at Alkaril Chemicals Inc., Henkel Corp. and Mona Industries. He has been involved in the personal care market for more than 30 years.

$\mathrm{O}$ 'Lenick has received a number of awards for his work in chemistry. He is a fellow of the Society of Cosmetic Chemists and was a member of their Committee on Scientific Affairs. He and is currently a member of the society's Education Committee.

$\mathrm{O}^{\prime}$ Lenick is the author of two books, Surfactants Chemistry and Properties, published in 1999, and Silicones for Personal Care, published in 2003 . He teaches continuing education courses in silicones and patent law for the SCC. He has also published more than 45 technical articles in trade

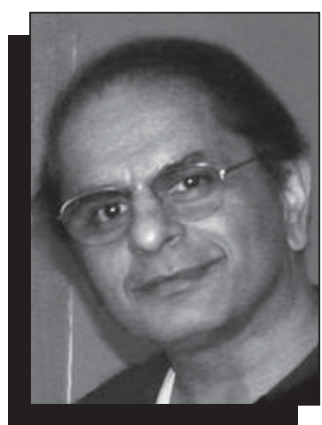

Manoj Misra

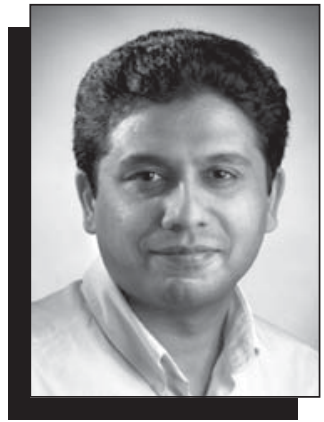

Kumar Subramanyan

journals, contributed chapters to five books, and is the inventor on over 270 patents.

O'Lenick can be reached by e-mail at tolenick@ mindspring.com.

Kevin A. O'Lenick is the vice president of sales and marketing for SurfaTech Corporation in Dacula, Ga. Founded in 1997, SurfaTech is a specialty chemical company that represents several companies, including Siltech

LLC, in the southeastern

U.S. and has its own line of specialty chemicals.

O'Lenick is the inventor on seven U.S. patents.

O'Lenick can be reached by e-mail at kevin@surfatech.com.

Linda D. Rhein is an adjunct professor for the master's in cosmetic science program at Fairleigh Dickinson University in Madison, N.J. She also is

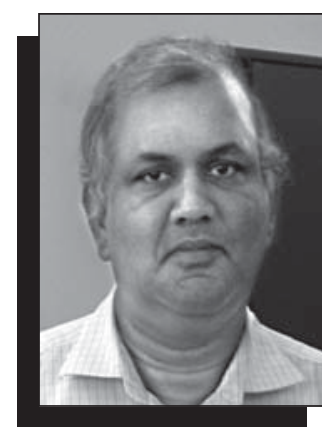

Surajit Mukherjee

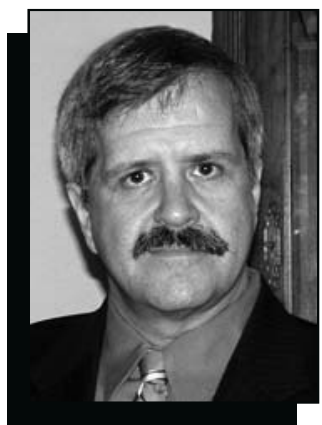

Anthony J. O'Lenick

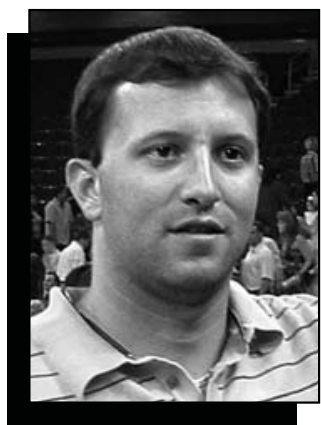

Kevin A. O'Lenick employed at Bayer USA as deputy director of over-the-counter drug safety. She received her MS and PhD degrees in biochemistry at the University of Maryland and did postdoctoral research at the

University of

Pennsylvania.

Rhein has 25 years of industry experience in research and development in cosmetic science, personal care, feminine care, and pharmaceutical research in dermatology. She has achieved global recognition for research in skin lipid biophysical structure and also mechanisms of surfactant damage of skin. Rhein has received numerous awards, the most prestigious being the Society of Chemists' Literature Award and Merit Award. She also has been elected president of the Society of Cosmetic Chemists. Rhein has published more than 50 scientific articles and presented more than 25 invited lectures in her field.

Rhein can be reached by e-mail at linda.rhein@ bayer.com.

P. Somasundaran is the LaVon Duddleson Krumb Professor of Mineral Engineering at
Columbia University's School of Engineering and Applied Science and also is director of the Langmuir Center for Colloids and Interfaces. Somasundaran received his BS degree from Kerala University in India, his BE degree from the Indian Institute of Science in Bangalore, and his MS and PhD degrees from the University of California, Berkeley. His research interests include surface and colloid chemistry; nanoparticles; selfassembly at interfaces; interfacial molecular interactions using advanced spectroscopy; polymer/surfactant adsorption dispersion, and deposition; wettability; biosurfaces; and environmental engineering. Somasundaran is a member of the U.S. National Academy of Engineering and the equivalent academies in China, India, and Russia. $\mathrm{He}$ is the recipient of many awards including the Ellis Island Medal of Honor, and AIME's Education, Richards, Gaudin, and Taggart Awards. Somasundaran also has authored and co-authored more than 500 publications.

Somasundaran can be reached at Columbia University, 905 Mudd Building, 500 W. 120 St.,
New York, NY 10027, USA; tel. 212-854-2926, fax 212-854-8362, and e-mail ps24@columbia. edu.

Kumar Subramanyan is senior development manager of clinical research and claims development at Unilever Home and Personal Care in Trumbull, Conn. Subramanyan received MS and PhD degrees in surface and colloid science at Columbia University in New York. He joined Unilever Research and Development in 1997 and has worked in the Skin Products Division of Unilever Home \& Personal Care for more than 10 years. He is the author or co-author of numerous book chapters and articles published in such journals as Cutis, Dermatologic Therapy, the International Journal of Cosmetic Science, Langmuir, and the Journal of Colloid $\mathcal{E}$ Interfacial Science.

Subramanyan's research interests include clinical and biophysical evaluation of skin care and skin cleansing products, dry skin and moisturization, sensitive skin, and surfactant-skin interactions.

He can be reached by e-mail at kumar. subramanyan@unilever. com. 